\title{
Analysis of Human Age Estimation Process
}

\author{
Husain Lakdawala ${ }^{1}$, Rehan Mastan ${ }^{2}$, Jibran Patel ${ }^{3}$, Er. Nazneen Pendhari ${ }^{4}$ \\ Student, Computer Engineering, MHSSCOE, Maharashtra, India ${ }^{1,2,3}$ \\ Professor, Computer Engineering, MHSSCOE, Maharashtra, India ${ }^{4}$
}

\begin{abstract}
Estimating human age automatically via facial image analysis has lots of potential real-world applications, such as multimedia communication, market intelligence and visual surveillance, and human computer interaction. However, it is still a challenging problem to automatically and effectively estimate human ages. Numerous researchers have worked on this interesting area and proposed different age estimation approaches. In this paper, we present an analysis on various age estimation techniques that were proposed in earlier literature. Also we provide an overview of the age estimation process.
\end{abstract}

Keywords: Automatic Brightness, Human Age Estimation, Face Recognition

\section{INTRODUCTION}

Human faces, as important visual cues, convey a related to our topic. Each of them have their separate view significant amount of nonverbal information to facilitate on this subject and different approaches accordingly. This the real-world human-to-human communication. As a section of the paper presents a survey on related papers result, the modern intelligent systems are expected to have that was proposed earlier.

the capability to accurately recognize and interpret human faces in real time. Facial attributes, such as identity, age, gender, expression, and ethnic origin, play a crucial role in real facial image analysis applications including multimedia communication, human computer interaction $(\mathrm{HCI})$, and security [1].

Although automatic image-based age estimation is an important technique involved in many real world applications, it is still a challenging problem to estimate human ages from face images. Fig. 1 shows some face images of two individuals with different ages [1]. The aging process is determined by not only the person's gene but also many external factors, such as health, living location, and living style. Males and females may also age differently.

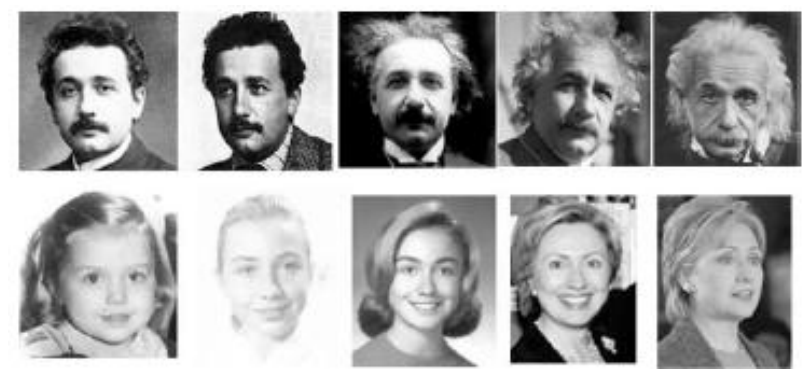

Fig. 1. Face aging of two individuals.

Each row shows images of the same individual at different ages

This paper presents an analysis on different age estimation techniques and an overview of available age estimation process.

\section{LITERATURE SURVEY}

We have performed extensive market research on age detection, as the main aim or the biggest hurdle in this project is to extract age from the facial image of the user. We have come across various API's and Technical Papers
Fares Alnajar, Caifeng Shan, Theo Gevers and Jan-Mark Geusebroek, July 2012 [2] has proposed a system which adopts a learning-based encoding method for age estimation under unconstrained imaging condition. There has been similar work done for face recognition in real life face images. They propose an approach to extract robust and discriminative facial features \& use soft encoding. The encoding is done by assigning each pixel to one of the multiple candidate code. And then the last step is to plot orientation histogram of local gradients.

Patch-based code learning is basically code set is learned from the whole face using the sampled vectors. From the sampled vector, a histogram is created. Once all the histogram is been created, they are all concatenated to form a global descriptor. Each human face is different, each of them has a different pattern, color and patched. Each individual can a different codes for a patch, consider an example like in a certain patch some of the code may appear in majority while for another patch the same set of codes are rare to be found. To illustrate this point they performed a certain experiment in which they took data set of 2000+ images and developed 2 code set from those images. Later when they extracted the sample vector from those images using these 2 code sets and constructed histogram of the same. The histogram is as follows -
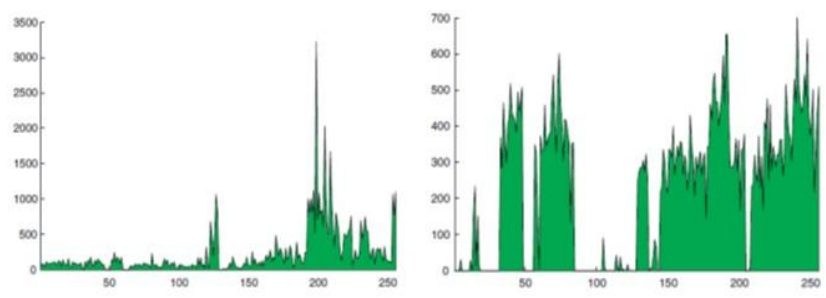

Fig. 2 shows the 2 histogram created using the sample vectors using the two code set over the $2000+$ images data set. 
Soft Encoding is the input image with the learned codebook, each sampled vector (at each pixel) is assigned to the closest code. This is known as Hard Coding. Soft encoding is used in image classification. In real world, for facial image, ambiguities will exists. In simple terms, consider a given sample vector, there is a possibility where there are more than one candidate codes. The assignment of code which is closest becomes difficult as the encoding becomes sensitive to image noise and varying conditions. These factors distort the sampled vector, which result in different code assignment. Hence to avoid this problem they have used the soft encoding assignment schemes which assigns the given sample vector to multiple codes with weights. Soft encoding is used in image classification.

Guodong Guo, Yun Fu, Charles R. Dyer, and Thomas S. Huang in July 2008 [1] introduced the new learning scheme based on age manifold learning for extracting age along with other facial features. It also designs a locally adjusted robust regressor for estimating ages of the human in question. The authors also claim that this approach increases the accuracy of age estimation by significant number as compared to previous methods. Basically, there are 3 main categories that can classify most existing age estimation methods. These are anthropometric model, age regression, and aging pattern subspace. The facial skin wrinkle analysis is basically used to create the anthropometric model. The face can be categorized into various age groups by the changes in the face shape, texture pattern related to growth and many other such factors. But the problem is this method is most suitable or convenient for young people, However they are not designed for refined age estimation and classification.

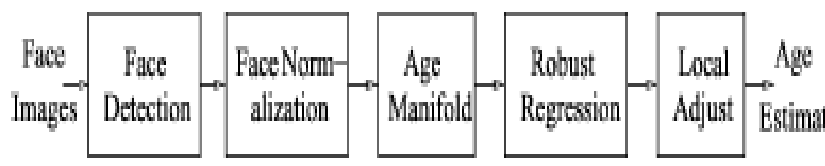

Fig. 3 Age estimation framework based on face image analysis and statistical learning.

The first step includes detecting and cropping the face part from the entire image, this process is known as face detection. Here we collect a large number of images from different age group and test accordingly. Now in this step, we take the cropped face patch from the previous step and the perform normalization which includes geometric alignments and illumination normalization. This is basically histogram equalization. Age Manifold is used to learn to map the face image into a low dimension. Basically we transform the face image into a sub space which is of low dimension. This is now provided as the input to the next stage Robust Regression function. In Robust Regression, basically a function which is developed is applied to the Age Manifold data. This function simply does the function of fitting the Age Manifold data. In other words function is applied to fit the
Age Manifold data. The last step in the process is local adjustment. The adjustment is done of the result of regression which is generated in the previous step. This step is performed to refine the local fitting of the data. So we can describe the working in short using an example. Consider a face image; the face image goes through the process of face detection followed by face normalization. After normalization, the normalized face image is project on the Age Manifold learning which has been already computed earlier during learning stage. Than after Manifold learning comes the final step where actual age of the face image is predicted using robust regression function.

Chin-Teng Lin, Dong-Lin Li, Jian-Hao Lai, Ming-Feng Han and Jyh-Yeong Chang, 29 Aug 2012 [3] introduced a novel and reliable framework is proposed for automatic age estimation. The age estimation is based on computer vision. Additionally, the proposed system can extract features from face image and estimate the age of the same in real time. So this unique feature has more potential in various application as compared to traditional semiautomatic systems. The result generated from this novel approach could be widely used as a real world application in various domains.

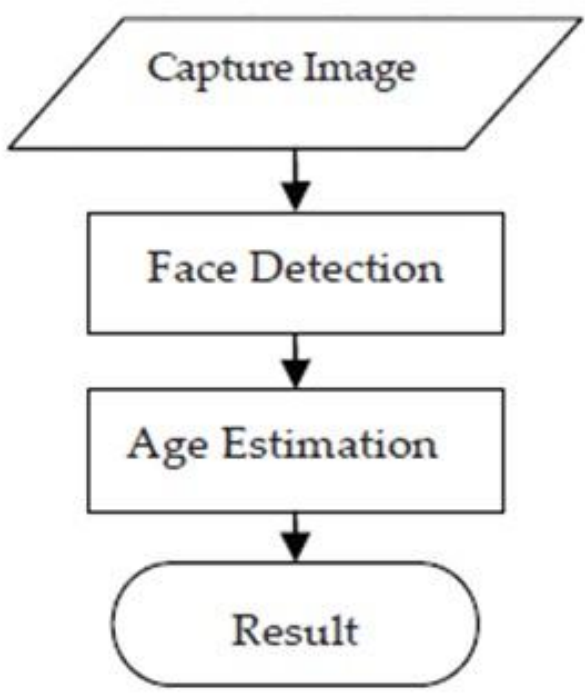

Fig. 4 System Overview

The figure 3 shows the basic and simple age estimation model along with basic step to achieve the same. The system proposed in this paper consists of 2 major parts or modules. Face Detection and Age Estimation. We will see these 2 modules in details. Face Detection localizes the facial region in the image. Here different size of window is applied to the image. The basic aim of doing so is to search the multi-scale facial candidates due to the distance between the camera and the object or person in question. There are basically 12 block searching windows of different size, as mentioned earlier these window search for multi-scale purpose and the size of windows ranges from smallest $(24 \times 24)$ with scaling factor of 1.25. The first step in Face Detection is Lighting 
Normalization, which is based on histogram method. The main aim of histogram fitting method is to convert or transform the $\mathrm{H}(\mathrm{l})$ \{original histogram \} to $\mathrm{G}(\mathrm{l})$ \{result or target histogram $\}$. The $\mathrm{G}(\mathrm{l})$ is estimated as the histogram of the image closest to the mean value which is present in the face database.

$$
\mathrm{M}_{\mathrm{H}->\mathrm{G}}(\mathrm{l})=\mathrm{M}_{\mathrm{U}-\mathrm{G}}\left(\mathrm{M}_{\mathrm{H}->\mathrm{U}}(\mathrm{l})\right)
$$

Where $\mathrm{M}_{\mathrm{H}->\mathrm{U}}(1)$ and $\mathrm{M}_{\mathrm{H}-\mathrm{G}}(1)$ are basic histogram mapping or they can also be called as the inverse mapping from $\mathrm{H}(\mathrm{l})$ and $\mathrm{G}(\mathrm{l})$ which transforms the histogram into uniform distribution respectively.

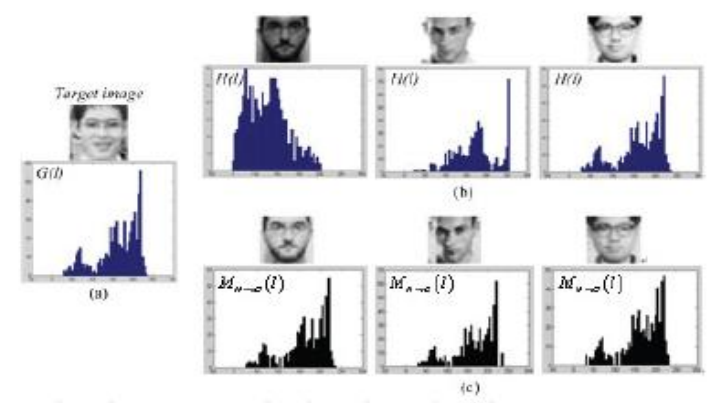

Fig 5 Lighting Normalization

Target Image (b) Input Images (c) Lighting Normalization Images.

In this paper the intensity based feature that is employed were based on Haar features. There are basically four type's rectangular features as shown in figure 5 . The four features are the vertical edge, horizontal edge, vertical line and last diagonal edge.
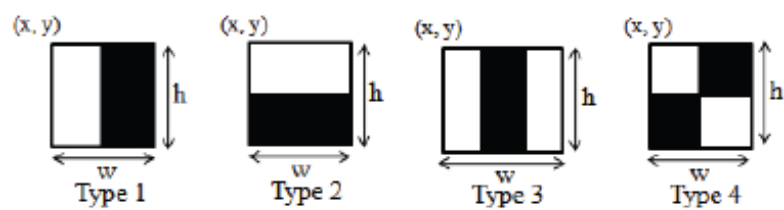

Fig. 6 Rectangular Features

The feature can be define by -

$$
\text { valve }_{\text {subtracted }}=f(x, y, w, h, \text { type })
$$

where $(\mathrm{x}, \mathrm{y})$ is the origin of coordinate of rectangular feature inside the search window. The $\mathrm{w}$ and $\mathrm{h}$ states the width and height of rectangular feature respectively. Type denotes the type of rectangular feature to be used and valve subtracted is basically the difference between the sum of pixel in white rectangle and sum of pixels in the dark rectangle. Rectangular feature which separates the face and non-face samples can be considered as the weak classifier. The weak classifier is denoted by $\mathrm{h}(\mathrm{x}, \mathrm{f}, \mathrm{p}, \theta)$ and it is given by equation -

$$
\begin{aligned}
\mathrm{h}(\mathrm{x}, \mathrm{f}, \mathrm{p}, \theta) & =1, \text { if } \mathrm{pf}(\mathrm{x})<\mathrm{p} \theta \\
& =0, \text { otherwise }
\end{aligned}
$$

During face detection, there is normally a case where more than one face candidate is detected even if there is exactly one face in the image. To avoid this problem we use Region Based Clustering.
Age Estimation contains 3 steps in this age estimation system, age feature extraction, feature reduction and feature classification. The age feature extraction is performed using Gabor wavelets. The Gabor wavelet is represented using convolution of the image with family of Gabor kernels. The scope of Gabor wavelet feature space is very high. Therefore it is very much important to select the most relevant features and to further reduce the dimension. The Gabor wavelet features are used in the SVM classifier to determine the age of the face. Support Vector Machines (SVM) has a good amount of potential to classify the sparse training data as they are developed to solve classification.

\section{CONCLUSION}

This paper presents an analysis on age estimation techniques and different frameworks of age estimation process. Age estimation is a challenging and interesting task. Different approaches like the locally adjusted robust regressor (LARR) method give better age estimation and outperform many state-of-the-art approaches to age estimation. The learning-based encoding method extracts and learns multiple codebooks for individual face patches instead of learning the set of codes for entire face. The Gabor wavelet transform achieves fully-automatic and real-time age estimation.

\section{REFERENCES}

[1] Guodong Guo, Yun Fu, Charles R. Dyer, and Thomas S. Huang, "Image-Based Human Age Estimation by Manifold Learning and Locally Adjusted Robust Regression" - IEEE Transactions On Image Processing, Vol. 17, No. 7, July 2008

[2] Fares Alnajar, Caifeng Shan, Theo Gevers and Jan-Mark Geusebroek, "Learning-based encoding with soft assignment for age estimation under unconstrained imagingconditions". - Image and Vision Computing, July 2012

[3] Chin-Teng Lin, Dong-Lin Li, Jian-Hao Lai, Ming-Feng Han and Jyh-Yeong Chang, "Automatic Age Estimation System for Face Images" International Journal of Advanced Robotic Systems, 29 Aug 2012.

[4] Eran Eidinger, Roee Enbar and Tal Hassner, "Age and Gender Estimation of Unfiltered Faces" - IEEE Transactions on Information Forensics and Security, 2013.

[5] Sven Ewan Shepstone, Member IEEE, Zheng-Hua Tan, Senior Member IEEE and Søren Holdt Jensen, Senior Member IEEE,"Audio-based Age and Gender Identification to Enhance the Recommendation of TV Content" - Audio-based Age and Gender Identification to Enhance the Recommendation of TV Content IEEE 2013.

[6] Xin Geng, Zhi-Hua Zhou, Senior Member, IEEE, and Kate SmithMiles, Senior Member, IEEE,"Automatic Age Estimation Based on Facial Aging Patterns" - IEEE Transactions On Pattern Analysis And Machine Intelligence, Vol. 29, No. 12, December 2007. 